\title{
Patient-Centered Rounding in an Inpatient Pediatric Setting
}

\section{Judy C. Stribling, Keith C. Mages \& Diana Delgado}

To cite this article: Judy C. Stribling, Keith C. Mages \& Diana Delgado (2017) Patient-Centered Rounding in an Inpatient Pediatric Setting, Journal of Hospital Librarianship, 17:2, 97-105, DOI: 10.1080/15323269.2017.1291047

To link to this article: http://dx.doi.org/10.1080/15323269.2017.1291047

$$
\text { 曲 Published online: } 14 \text { Apr } 2017 .
$$

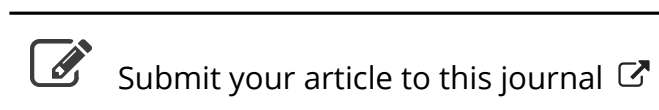

Llll Article views: 38

Q View related articles $\sqsubset$

View Crossmark data ¿ 


\title{
Patient-Centered Rounding in an Inpatient Pediatric Setting
}

\author{
Judy C. Stribling, Keith C. Mages, and Diana Delgado \\ Samuel J. Wood Library, Weill Cornell Medicine, New York, New York, USA
}

\begin{abstract}
Clinical Medical Librarians at Weill Cornell Medicine (WCM), Samuel J. Wood Library provide unique health literacy services for patients, families, and caregivers on the Phyllis and David Komansky Center for Children's Health pediatric floors of New York-Presbyterian Hospital (NYPH) by conducting twice weekly patient rounds and bringing consumer health information and services to bedsides. Although in its infancy, the Pediatric Consumer Librarian Service (PCLS) generates statements of appreciation from patients and parents, enthusiasm among stakeholders, and reports of increased job satisfaction by librarians. We report on experiences, methods, feedback from users, and plans for the future.
\end{abstract}

\section{ARTICLE HISTORY}

Received 28 October 2016

Accepted 29 December 2016

\section{KEYWORDS}

Consumer health; hospital patients; inpatients; pediatrics; rounds

\section{Background}

Librarians and clinicians in the Department of Pediatrics at NYPH have a long-standing working relationship. For over 10 years, librarians have participated in weekly clinical rounds with attendings and residents, and have subsequently been involved in teaching evidence-based medicine to medical students in their first year and during third-year pediatric clinical rotations. This close relationship led to a year-long period between 2012 and 2013 when a WCM librarian-who was also the Manager of the college-supported Myra Mahon Patient Resource Center (PRC), a consumer health library located across the street from the hospital-provided consumer health support for patients and parents on the pediatric floor. Unfortunately, the pediatric consumer health program ended when the librarian left the institution.

Between the years 2013 and 2016, the WCM library increased library faculty by $33 \%$. This increase included the onboarding of a new Assistant Director of Clinical Services who also serves as Manager of the PRC (Assistant Director), adding an additional Clinical Medical Librarian, and initiating a Clinical Medical Library internship program which brought a

CONTACT Judy Stribling jcs2002@med.cornell.edu 0 Samuel J. Wood Library, Weill Cornell Medicine, 1300 York Avenue, New York, NY 10065, USA.

Color versions for one or more of the figures in this article can be found online at www.tandfonline.com/whos.

Published with license by Taylor \& Francis $\odot$ Judy C. Stribling, Keith C. Mages, and Diana Delgado 
full-time library student (Intern) onto the team. The addition of personnel allowed leadership to review and assess the value of creating new programming, tweaking existing services, and reviving defunct programs. The PCLS was launched based upon the following considerations:

- many parents and family members of hospitalized pediatric patients are vigilant caregivers unwilling to leave the bedside of their loved ones to seek information (1)

- low health literacy remains a problem in the United States yet systematic reviews of health literacy research suggest health literacy interventions are associated with improvements in clinical outcomes (2-5)

- low health literacy has special detrimental implications for children because they are dependent upon parents and caregivers (6)

- health literacy, similar to information literacy, requires people of all ages and educational levels to acquire additional skills such as informationseeking behavior $(7,8)$

- health literacy is a dynamic issue that requires system-level changes for health professionals and organizations (9)

- while health care providers may not be the most effective literacy communicators, librarians are almost ideally suited for the job $(10,11)$

Plans to resume and improve consumer support for pediatrics began in earnest in June 2016, during a casual conversation between the library's Assistant Director and the Department of Pediatric's Chair of Quality and Patient Safety. Following that conversation, the PCLS team met and defined pediatric consumer support mission goals, identified key clinical stakeholders, and developed operational and marketing strategies. Informed of the PCLS team's progress, the Chair formalized the Pediatric Department's request for consumer health support services at the end of June 2016. The newly formed team began conducting consumer health rounds on August 9, 2016.

\section{Literature review}

Library and biomedical databases were searched for articles about librarians performing consumer health rounds at patient bedsides. The research revealed a plethora of information about librarians conducting clinical rounds but little about consumer rounds despite the fact that consumer health librarians ranked patient bedside delivery of health information as one of the most important outreach services they provided (12). No article was discovered about consumer health rounding by librarians designed specifically for pediatric patients and their families. The article most closely related to the PCLS program was by Cleo Pappas, a clinical medical librarian 
at the Children's Hospital of University of Illinois, who described her experience rounding with pediatric physicians and introducing iPads to the service (13).

Experiences at several institutions with general patient populations informed the development of the new PCLS program. Among the most notable was an overview of a service developed at Englewood Hospital and Medical Center (14). Especially valuable was the description of Englewood librarians' introductory script and on-unit procedures. Similarly, a program within the Aurora Health Care system reminded the PCLS team to pay strict attention to infection control, patient reading levels, and patient-preferred method of information delivery (15). Both articles mentioned the importance of communicating with nurses-a sentiment the PCLS team shared from the project's onset. Finally, Vanderbilt's Patient Information Consult Service provided useful guidance for development of consumer health information packets (16). Articles from an additional biomedical literature search reinforced the importance patients place on educational handouts. This strengthened the decision the team made to provide printed materials whenever possible $(17,18)$.

\section{Planning}

Development of the PCLS began with the formation of a team consisting of the Associate Director of User Support, Research and Education, the Assistant Director, a Clinical Medical Librarian, and Clinical Medical Library Intern (Intern). The team held a brainstorming session where it defined the program's mission and goals, identified key clinical stakeholders - physicians, nurses, social workers, and the hospital's Child Life staff - and drafted an initial service overview. The primary mission of the PCLS is to provide pediatric patients and their family members with access to reliable, targeted, and timely health information resources that encourage and enable informed decision making. Secondarily, the team seeks to improve patient satisfaction and comfort during their hospital stay. Goals of the program included returning health information on the same day requested, marketing PRC and medical library services, and reinforcing the message Amazing Things Are Happening for Kids at NYPH.

Initially, the team planned to provide bedside rounding on the main pediatric floor answering questions in real time, or developing targeted information packets to be printed and delivered to the bedside, by e-mail, or U.S. mail, depending upon patient preference. Additional librarian-inresidence hours at the family lounge were planned. This would provide a period of time for interested patients or family to visit with a librarian to discuss questions that arose before or after information rounds. The PCLS 
team generated a list of procedures to address issues based on librarian's clinical rounding and consumer health experience.

Once goals and services were identified, the team requested introductions from the Chair to other clinical stakeholders within the pediatric department. The Chair quickly responded and arranged meetings between the PCLS team and Patient Care Directors (PCDs) from the General Pediatric Unit (Peds), the Pediatric Intensive Care Unit (PICU), and the Neonatal Intensive Care Unit (NICU). The PCDs, who are members of nursing management responsible for overseeing the clinical activities of each of the hospital's pediatric units, were receptive and enthusiastic about the new service. The PCD of the Peds Unit made several suggestions including that a good time to conduct rounds would be between 1:00-3:00 PM, because this is a quiet time when the playroom is closed and clinical procedures and medication administrations are kept to a minimum. The PCD also suggested the team develop a series of information packets targeted for pediatric cancer patients citing a growing need for this information.

Meetings with the intensive care PCDs were equally productive and led to unexpected request for rounding on the PICU floor and the possibility of conducting patient education research with NICU nurses. All three PCDs agreed to inform staff nurses about the PCLS program and share their support for the service.

\section{Procedures}

PCLS team members are required to wear white lab coats, which identify them by name and title during all on-floor patient encounters and Weill Cornell Medicine security badges while on hospital property. Team members observe all infection control and safety precautions when entering a patient's room. PCLS team members must abide by NYPH vaccination policies.

Acting upon the suggestion of the Peds PCD, PCLS rounds are scheduled on Tuesdays and Thursdays during the quiet hour between 1:00 and 2:00 PM. At least two of the three team librarians, occasionally accompanied by the Intern, begin rounding on the 30-bed general Peds floor and finish visiting patients on the 50-bed PICU. The Intern maintains in-residence hours at the family lounge each Wednesday and Thursday between 1:30 and 2:30 PM. The PCLS team's schedule, made available across both pediatric units, is posted in a colorful brochure complete with team picture, contact information, and a description of the program.

On the general Peds floor, librarians check in at the nursing station at the beginning of each rounds session to inquire if any patients or families on unit have specific information needs. PCLS team members also inquire if the nurses have evidence-based practice questions and encourage them to utilize the librarian's skills and resources. If a patient with a health question is 
identified, he or she is visited first. Otherwise, the team rounds every room except those where a patient is clearly at rest or an occasional clinical procedure is being performed. Upon entering a patient's room the team engages with both the patient and present family members by loosely following the script:

Hello, we just wanted to take a minute of your time to introduce ourselves: My name is and this is We're consumer health librarians. We help patients and their families get health information that is easy to read andunderstand and trust. It can be information about your current diagnosis, treatment, or any other health or wellness questions you might have.

Rounding procedures in the PICU are different from general Peds for a number of reasons. PICU patients are critically ill, the unit has no official quiet time, and each room contains multiple beds with an individual nursing station positioned at the entrance of each room. The team checks in with nurses at each station unless it appears a patient is in crisis or a clinical procedure is being performed. Nurses refer PCLS team members to patients or families they believe may have information needs and to those whose health status is stable enough for a visit. PICU nurses are also encouraged to ask evidence-based practice questions at this time.

The PCLS team developed a HIPAA compliant request log with no identifying patient data other than room number to track patient information inquiries. The form has fields for the date of information request, room number, patient's question, information medium or delivery method, and finally a field to check once the question has been answered and information delivered.

Patients may opt to have information delivered by print at bedside, by e-mail, or U.S. mail. PCLS team members make every effort to deliver information the same day but no later than 24 hours from request. All patient inquiries are made in confidence. If a patient asks questions that may affect clinical care, librarians ask for the patient's permission to share the information with the clinical care team.

PCLS librarians use a variety of sources to answer consumer health questions. The most used resources are: Access Pediatrics, Clinical Key Patient Education, Medline Plus, MICROMEDEX Care Notes, and UpToDate Patient Education resources. PCLS team members search for the most appropriate pediatric consumer health information to share with patients. Because many innovative products or interventions are not readily available for the pediatric population, there are gaps in pediatric health research and scientific literature which affect the availability of appropriate of appropriate consumer health information (19). When pediatric specific information for a patient or parent's question is unavailable, PCLS librarians create a summary of evidence from the best available health resources (16). 


\section{Program stats and evaluation (results)}

Between August 9, 2016 and October 27, 2016, the PCLS team conducted 24 rounds on the general Peds and PICU floors and answered 94 patient, family, or health-provider questions. Then, 139 health information items were delivered in a variety of formats described in Table 1.

Questions fell into six broad categories detailed in Figure 1.

Anecdotal evidence suggests the PCLS program satisfies the needs of patient's family members. One mother, concerned about her 12-year-old son receiving narcotic pain medication, asked PCLS team members about pediatric morphine dosing. When asked if the information she received was informative she replied, "Yes, it was very helpful. I wasn't happy about him having to take that medication, but if he needs it, I'm OK. I feel much better about it now."

Team librarians develop familiarity with patients requiring long or repeated hospital stays. One long-stay patient's mother relied heavily on the PCLS team to augment information about her son's difficult

Table 1. Mode and Means of Information Delivery.

\begin{tabular}{lr}
\hline Medium & \multicolumn{1}{c}{ Number } \\
\hline Printed material delivered at bedside & 127 (13 in Spanish Language) \\
Printed material sent via U.S. mail & 2 \\
Electronic material sent via e-mail & 7 \\
Videos displayed at bedside & 1 \\
Books loaned to patient & 2 \\
TOTAL & 139 \\
\hline
\end{tabular}

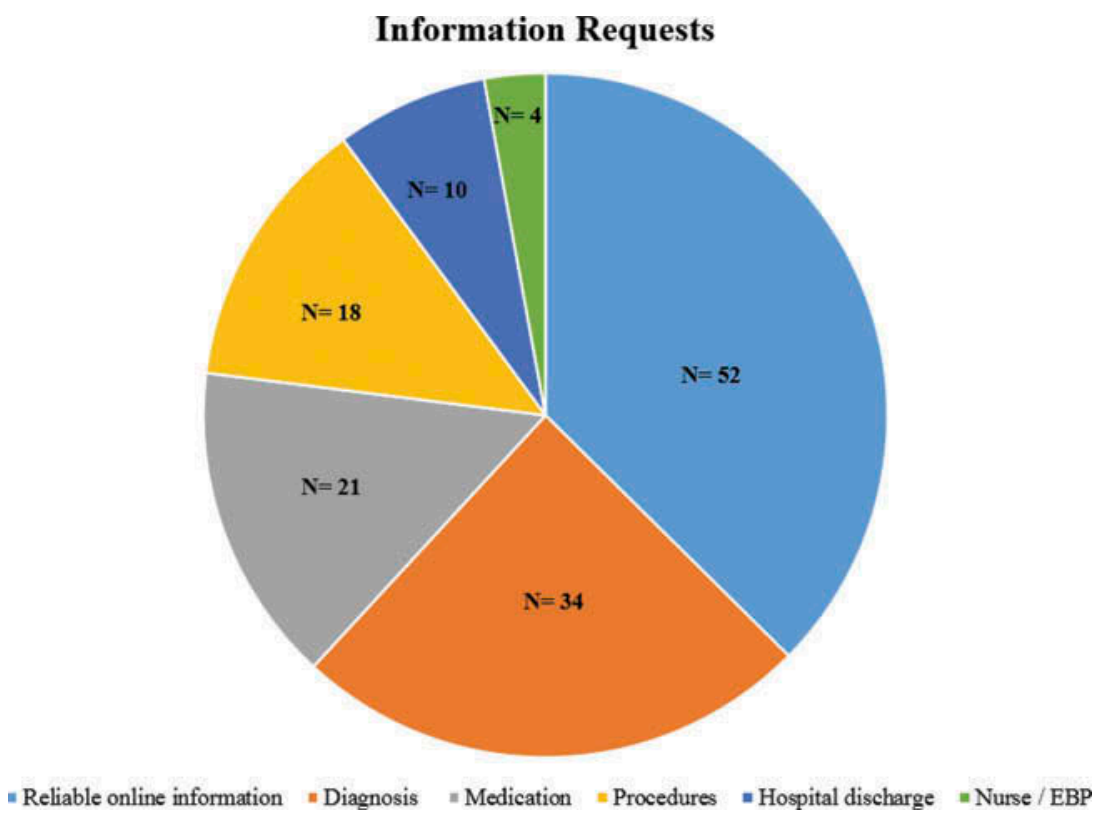

Figure 1. Information requests by topic. 
diagnosis and credited the librarians for "making us all feel a little more in control."

Told he could contact the team after hospital discharge, a father said, "For parents at home, information is everything. This is great."

Clinicians appear equally pleased with services provided by the librarians. The PCD of the PICU wrote to the Weill Cornell Medicine Library Director about the program:

Parents and staff have been thoroughly impressed with the service offered by the librarian team and very appreciative of the information they have provided. I am looking forward to working with them on evidence-based nursing quality improvement projects. Thank you for offering such a great service for our families and staff.

Other nurses have commented: What a wonderful service, this will be very helpful; Awesome idea!; and, I'm calling on you when I do my EBP project.

One PICU pediatrician contacted a PCLS team member on a nonrounding day to ask if the team might consult with her patient.

\section{Discussion and next steps}

The program's success is partially demonstrated by usage statistics and observed anecdotal comments, however, a formalized evaluation tool is not currently utilized. The PCLS team plans to develop survey tools to measure the program's impact upon patient satisfaction and knowledge retention. A separate tool to measure satisfaction among clinicians is also being considered. Formal program evaluation will be undertaken at 6 and 12 months, and the program adjusted accordingly.

Connecting with preteen pediatric patients has been one of the most difficult tasks faced by the team. The Assistant Director plans to purchase children's books and coloring books related to being in the hospital, having an illness, and fostering self-worth. Subject and coloring books will be made available to young patients during the Intern's onunit hours at the family lounge. Coloring books will become part of the PCLS librarian's rounding toolkit.

\section{Conclusion}

Pediatric units possess distinct policies and cultures and the PCLS program is tailored for two very specific micro-climates at NYPH. Therefore, the PCLS program may not be generalizable to other pediatric hospitals. However, the PCLS team encourages librarians interested in pediatric patient rounding to 
engage with physicians, nurses, and (when applicable) child life specialists to develop similar services.

\section{References}

1. Dudley SK, Carr JM. Vigilance: the experience of parents staying at the bedside of hospitalized children. J Pediatr Nurs. 2004;19(4):267-275.

2. Sheridan SL, Halpern DJ, Viera AJ, Berkman ND, Donahue KE, Crotty K. Interventions for individuals with low health literacy: a systematic review. J Health Commun. 2011;16(Sup3):30-54.

3. Berkman ND, Sheridan SL, Donahue KE, Halpern DJ, Viera A, Crotty K, Holland A, Brasure M, Lohr KN, Harden E, Tant E, Wallace I, Viswanathan M. Health literacy interventions and outcomes: an updated systematic review. Evid Rep Technol Assess. 2011; (199):1-941.

4. Jacobs RJ, Lou JQ, Ownby RL, Caballero J. A systematic review of eHealth interventions to improve health literacy. Health Inform J. 2016;22(2):81-98.

5. Taggart J, Williams A, Dennis S, Newall A, Shortus T, Zwar N, Denney-Wilson E, Harris MF. A systematic review of interventions in primary care to improve health literacy for chronic disease behavioral risk factors. BMC Fam Pract. 2012;13:49.

6. Gebhard RD, Goske MJ, Salisbury SR, Leopard AC, Hater DM. Improving health literacy: use of an informational brochure improves parents' understanding of their child's fluoroscopic examination. AJR Am J Roentgenol. 2015;204(1):W95-W103.

7. Rothman RL, Yin HS, Mulvaney S, Co JP, Homer C, Lannon C. Health literacy and quality: focus on chronic illness care and patient safety. Pediatrics. 2009;124 Suppl 3:S315-326.

8. Arndt TS, Watstein SB. Health literacy: a natural role for librarians. Ref Serv Rev. 2016;44(2):81-84.

9. Koh HK, Berwick DM, Clancy CM, Baur C, Brach C, Harris LM, Zerhusen EG. New federal policy initiatives to boost health literacy can help the nation move beyond the cycle of costly 'crisis care'. Health Aff (Millwood). 2012;31(2):434-443.

10. Ha JF, Longnecker N. Doctor-patient communication: a review. Ochsner J. 2010;10 (1):38-43.

11. Shipman JP, Lake E, Weber AI. Improving health literacy: health sciences library case studies. Ref Serv Rev. 2016;44(2):206-214.

12. Oelschlegel S, Gonzalez AB, Frakes E. Consumer health information centers in medical libraries: a survey of current practices. J Hosp Librariansh. 2014;14(4):335-347.

13. Pappas C. Medical librarian rounding with an iPad. Libr Technol Rep. 2012;48 (8):22-27.

14. Linder KL, Sabbagh L. In a new element: medical librarians making patient education rounds. J Med Libr Assoc. 2004;92(1):94-97.

15. Strube K, Hoffmann ML, Melchiors A, Egebo H, Webb L. Patient information rounds in a hospital system. J Hosp Librariansh. 2006;6(4):13-28.

16. Williams MD, Gish KW, Giuse NB, Sathe NA, Carrell DL. The Patient Informatics Consult Service (PICS): an approach for a patient-centered service. Bull Med Libr Assoc. 2001; 89(2):185-193.

17. Gupta RS, Shuman S, Taveras EM, Kulldorff M, Finkelstein JA. Opportunities for health promotion education in child care. Pediatrics. 2005;116(4):e499-505. 
18. Wahl H, Banerjee J, Manikam L, Parylo C, Lakhanpaul M. Health information needs of families attending the paediatric emergency department. Arch Dis Child. 2011;96 (4):335-339.

19. Connor E, Lombardi D, van den Anker J. More than baby steps: perspectives on pediatric translational research. Sci Transl Med. 2009;1(2):2cm2. 